\title{
The Relationship Between Synchronization Among Neuronal Populations and Their Mean Activity Levels
}

\author{
D. Chawla \\ E. D. Lumer
}

K. J. Friston

Wellcome Department of Cognitive Neurology, Institute of Neurology, London WC1N 3BG, U.K.

In the past decade the importance of synchronized dynamics in the brain has emerged from both empirical and theoretical perspectives. Fast dynamic synchronous interactions of an oscillatory or nonoscillatory nature may constitute a form of temporal coding that underlies feature binding and perceptual synthesis. The relationship between synchronization among neuronal populations and the population firing rates addresses two important issues: the distinction between rate coding and synchronization coding models of neuronal interactions and the degree to which empirical measurements of population activity, such as those employed by neuroimaging, are sensitive to changes in synchronization. We examined the relationship between mean population activity and synchronization using biologically plausible simulations. In this article, we focus on continuous stationary dynamics. (In a companion article, Chawla (forthcoming), we address the same issue using stimulus-evoked transients.) By manipulating parameters such as extrinsic input, intrinsic noise, synaptic efficacy, density of extrinsic connections, the voltage-sensitive nature of postsynaptic mechanisms, the number of neurons, and the laminar structure within the populations, we were able to introduce variations in both mean activity and synchronization under a variety of simulated neuronal architectures. Analyses of the simulated spike trains and local field potentials showed that in nearly every domain of the model's parameter space, mean activity and synchronization were tightly coupled. This coupling appears to be mediated by an increase in synchronous gain when effective membrane time constants are lowered by increased activity. These observations show that under the assumptions implicit in our models, rate coding and synchrony coding in neural systems with reciprocal interconnections are two perspectives on the same underlying dynamic. This suggests that in the absence of specific mechanisms decoupling changes in synchronization from firing levels, indexes of brain activity that are based purely on synaptic activity (e.g., functional magnetic resonance imaging) may also be sensitive to changes in synchronous coupling. 


\section{Introduction}

This article is about the relationship between fast dynamic interactions among neuronal populations and measures of neuronal activity that are integrated over time (e.g., functional neuroimaging). In particular, we address the question, "Can anything be inferred about fast coherent or phasic interactions based on averaged macroscopic observations of population activity?" This question is important because a definitive answer would point to ways in which data from functional neuroimaging might be related to electrophysiological findings, particularly those based on multiunit electrode recordings of separable spike trains.

The basic hypothesis behind this work is that fast dynamic interactions between two units in distinct populations are a strong function of the macroscopic dynamics of the populations to which the units belong. In other words, the coupling between the two neurons, reflected in their coherent activity over a time scale of milliseconds, cannot be divorced from the context in which these interactions occur. This context is determined by the population dynamics expressed over thousands of neurons and extended periods of time. More specifically, on the basis of previous theoretical and empirical work (Abeles, 1982; Aertsen \& Preissl, 1990; Lumer, Edelman, \& Tononi, 1997a, b), we conjectured that the degree of phase locking, or more generally synchronization, between units in two populations, would covary with the average activity in both populations. Our aim was to test this hypothesis using biologically plausible simulations over a large range of parameters specifying the physiological and anatomical architecture of the model. In this article we report simulations that address the relationship between mean activity and synchronization during relatively steady-state dynamics following the onset of continuous input lasting for a few seconds. (In a subsequent article, we will address the same issue using evoked transients and dynamic correlations at different levels of mean activity.)

Many aspects of functional integration and feature linking in the brain are thought to be mediated by synchronized dynamics among neuronal populations. In the brain, synchronization may reflect the direct, reciprocal exchange of signals between two populations, whereby the activity in one population affects the activity in the second, such that the dynamics become entrained and mutually reinforcing, leading to synchronous discharges. In this way, the binding of different features of an object may be accomplished, in the temporal domain, through the transient synchronization of oscillatory responses (Milner, 1974; von der Malsburg, 1981; Sporns, Tononi, \& Edelman, 1990). Physiological evidence has been generally compatible with this theory (Engel, Konig, Kreither, \& Singer, 1991). It has been shown that synchronization of oscillatory responses occurs within as well as between visual areas, for example, between homologous areas of the left and right hemispheres and between remote areas in the same hemisphere at different levels of the visuomotor pathway (Gray, Engel, Konig, \& Singer, 1990; Engel et al., 
1991; Konig, Engel, \& Singer, 1995; Roelfsema, Engel, Konig, \& Singer, 1997). Synchronization in the visual cortex appears to depend on stimulus properties such as continuity, orientation similarity and motion coherency (Gray, Konig, Engel, \& Singer, 1989; Engel, Konig, Kreiter, Gray, \& Singer, 1990; Freiwald, Kreiter, \& Singer, 1995). It would therefore seem that synchronization provides a suitable mechanism for the binding of distributed features of a pattern and thus contributes to the segmentation of visual scenes and figure-ground segregation. More generally, synchronization may provide a powerful mechanism for establishing dynamic cell assemblies that are characterized by the phase and frequency of their coherent oscillations. Accordingly, the effective connectivity among different populations can be modulated in a context-sensitive way by synchronization-related mechanisms. Taken together, these considerations indicate that synchronization is an important aspect of neuronal dynamics.

The aim of this study was to see if population synchrony bears some relationship to overall activity levels. We used physiologically based neuronal networks comprising two simulated brain areas to look at how the level of neuronal activity affects the degree of phase locking between the two populations and vice versa. We used two models. The first had a fairly realistic laminar architecture but simplified dynamics. The second had a simple architecture but detailed (Hodgkin-Huxley) dynamics. By modifying different parameters, such as synaptic efficacy, the density of extrinsic connections, the voltage-sensitive nature of postsynaptic mechanisms, the number of neurons, and the laminar structure within the neuronal populations, we were able to model a broad range of different architectures. For each architecture, we induced changes in the mean activity and synchronization among simulated populations by manipulating extrinsic input (or equivalently intrinsic noise). Analyses of the simulated spike trains and local field potentials showed that in almost all regions of the model's parameter space, mean activity and synchronization were tightly coupled.

\section{Methods}

2.1 Integrate and Fire Model. The first component of this study looked at the behavior of two reciprocally connected cortical areas. Each cortical area was divided into three laminae corresponding to the supra- and infragranular layers and layer 4 (see Figure 1a). This laminar organization is consistent with known cortical anatomy (Felleman \& Van Essen, 1991). Each layer contained 400 excitatory cells and 100 inhibitory cells. Intralaminar connections had a density of $10 \%$ and included both excitatory and inhibitory connections (with AMPA and GABAa synapses, respectively). The supragranular cells also expressed modulatory NMDA and slow GABAb synapses. The pattern of interlayer connections can be seen in Figure 1a. Interlaminar connections were $7.5 \%$ and excitatory. GABAb connections were also implemented from the supragranular layer to the other two layers to 
A

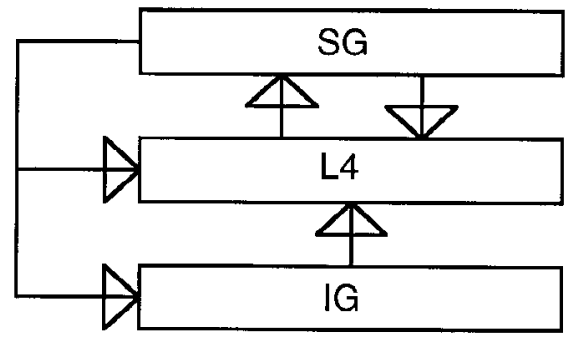

B

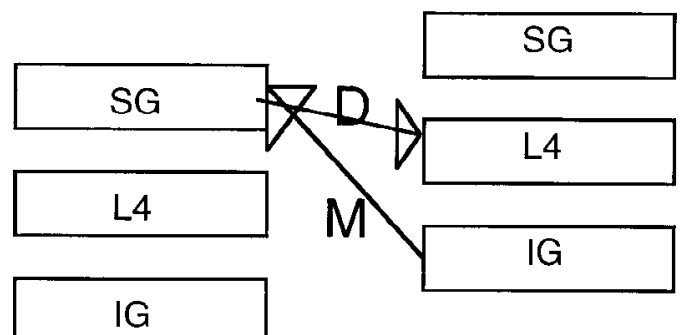

Figure 1: Architecture of the first model. (a) A schematic showing the connectivity structure within one cortical region. (b) Two cortical regions where the first cortical area provides driving input to the second, and the second cortical area provides modulatory input to the first. In these diagrams SG, L4, and IG refer to supragranular layers, layer 4 , and infragranular layers, respectively. D and $\mathrm{M}$ refer to driving (AMPA) and modulatory (NMDA) connections respectively.

represent double-bouquet cells (Conde, Lund, Jacobwitz, Baimbridge, \& Lewis, 1994; Kawaguchi, 1995). Our ratio of interlayer/intralayer connections approximated the $45 \% / 28 \%$ ratio reported in the cat striate cortex (Ahmed, Anderson, Douglas, Martin, \& Nelson, 1994).

Feedforward connections between cortical areas (see Figure $1 \mathrm{~b}$ ) were $5 \%$, from the supragranular excitatory cells in the first cortical area to the AMPA synapses of layer 4 cells in the second cortical area. Feedback connections were $5 \%$, from the infragranular excitatory cells of the second cortical area to the modulatory NMDA synapses of supragranular cells in the first cortical area. The synapse-to-neuron ratio in this model was consistent with experimental findings (Beaulieu \& Colonnier, 1983, 1985). The extrinsic, interareal connections were exclusively excitatory. This is consistent with known neuroanatomy where, in the real brain, long-range connections that traverse 
white matter are almost universally glutaminergic and excitatory. The excitatory extrinsic connections between the neuronal populations targeted both excitatory and inhibitory neurons within each population. These target neurons are randomly allocated to the excitatory afferent in proportion to the percentage of each cell type. This results in extrinsic connections targeting preferentially excitatory cells, which is consistent with the empirical data (Domenici, Harding, \& Burkhalter, 1996; Johnson \& Burkhalter, 1996). The anatomy used in this model was consistent with Lumer et al. (1997a) and has been tested against empirical data (Sukov \& Barth, 1998).

Individual neurons, both excitatory and inhibitory, were modeled as single-compartment, integrate-and-fire units (see the appendix, model 1). Synaptic channels were modeled as fast AMPA and slow NMDA for excitatory and fast GABAa and slow GABAb for inhibitory channels (Stern, Edwards, \& Sakmann, 1992; Otis \& Mody, 1992; Otis, Konick, \& Mody, 1993). These synaptic influences were modeled using dual exponential functions, with the time constants and reversal potentials taken from the experimental literature (see Lumer et al., 1997a, for the use and justification of similar parameters to those used in the present model). Adaptation was implemented in each excitatory cell by simulating a GABAb input from the cell onto itself. Adaptation is an important feature of neocortical cell behavior, and it has been observed consistently that repetitive cell stimulation produces a progressive and reversible decrease of spontaneous depolarizations and a decrease in firing rate (Calabresi, Mercuri, Stefani, \& Bernardi, 1990; Lorenzon \& Foehring, 1992). Implementing slow GABAb inhibitory inputs from each cell onto itself emulates this effect. Transmission delays for individual connections were sampled from a noncentral gaussian distribution. Intra-area delays had a mean of $2 \mathrm{~ms}$ and a standard deviation of $1 \mathrm{~ms}$ and interarea delays had a mean and standard deviation of $5 \mathrm{~ms}$ and $1 \mathrm{~ms}$, respectively. A continuous random noisy input was provided to all units in layer 4 of the first area. Variations in this input were used to induce changes in mean activity and synchronization.

2.2 Model Based on the Hodgkin-Huxley Formalism. Once we had characterized the relationship between phase locking and firing rate in the model above, we tried to replicate our results over a much larger parameter space within the framework of a simpler model consisting of two areas, each containing 100 cells that were $90 \%$ intrinsically connected. Due to the comparatively small number of cells used in this model, such a high connection density gives a similar synapse-to-neuron ratio as in the previous model. In this second component of our study, individual neurons were modeled as single-compartment units. Spike generation in these units was implemented according to the Hodgkin-Huxley formalism for the activation of sodium and potassium transmembrane channels. This facilitated a more detailed and biologically grounded analysis of effective membrane time constants (see below). Specific equations governing these channel dynamics can be 
found in the appendix (model 2). In addition, synaptic channels provided fast excitation and inhibition. These synaptic influences were modeled using exponential functions, with the time constants and reversal potentials for AMPA (excitation) and GABAa (inhibition) receptor channels specified as in the previous model. Cells were $20 \%$ inhibitory and $80 \%$ excitatory (Beaulieu, Kisvarday, Somogyi, \& Cynader, 1992). Reciprocal extrinsic (interarea) connections were all excitatory. Transmission delays for individual connections were sampled from a noncentral gaussian distribution, with means and standard deviations as given in the first model. A continuous random noisy input was provided to all units in one of the two areas (area 1). In some simulations, the mean interarea delay was increased to $8 \mathrm{~ms}$ to mimic a greater separation between the areas. In other simulations, excitatory NMDA synaptic channels were incorporated. These NMDA channels were used only in the feedback connections.

2.3 Data Analysis. The neuronal dynamics from both models were analyzed with the cross-correlation-function between time series from two areas, after subtraction of the shift predictor (Nowak, Munk, Nelson, James, \& Bullier, 1995). We used the time series of the number of cells spiking per millisecond (in each population) as well as the mean membrane potential or local field potential of each population. We ran the model for 2 seconds of simulated time, eight times. The cross-correlation between the first time series (eight runs in order) and a second time series, constituting eight runs in a random order, constituted our shift predictor. The shift predictor reflects phase locking due only to transients locked to the onset of each stimulation.

As a measure of the level of phase locking between the two populations, we used the peak cross-correlation following correction. This separates stimulus-related phase locking from that due purely to neuronal interactions, allowing us to see how phase locking due to the interactions between the two neuronal populations varied as a function of activity level.

The measure of phase locking given above is effectively a measure of the functional connectivity between the two areas. Functional connectivity has been defined as the correlation between two neurophysiological time series, whereas effective connectivity refers to the "influence" that one neuronal system exerts over another (Friston, 1994). In this work, we also examined how mean activity and phase locking vary with effective connectivity, using the second model. As our measure of effective connectivity, we used the probability (averaged over units and time) that a cell in the first population would cause a connected cell in the second population to fire. Furthermore, we tried to elucidate some of the mechanisms that could underlie the relationship between mean activity and synchronization in terms of temporal integration at a synaptic level. Our hypothesis was that high levels of activity would engender shorter membrane time constants. This, in turn, would lead to the selection of synchronized interactions by virtue of the reduced capacity for temporal integration (Bernander, Douglas, Martin, 
A

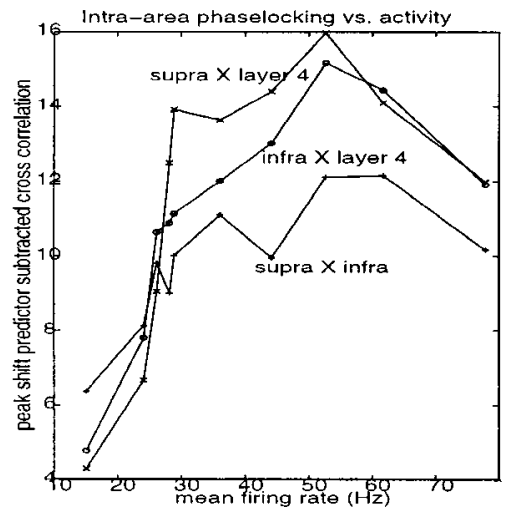

B

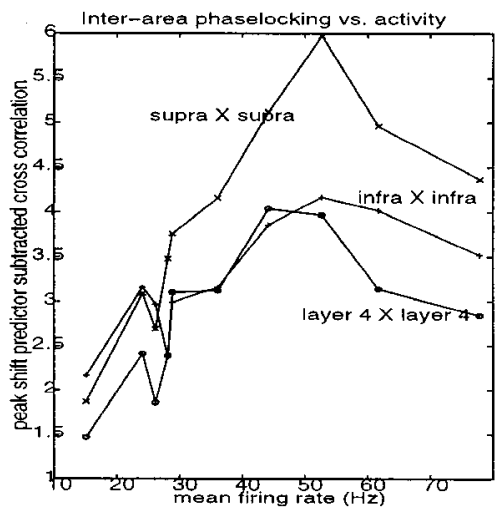

Figure 2: Synchrony versus mean activity for the first model. (a) A plot of the peak shift predictor subtracted cross-correlation between mean spike trains in different layers in area 1 of the first model against mean firing rate in population 1 , as the random input to population 1 was increased systematically. (b) A plot for the same input levels, but here the phase locking between homologous layers in each area is shown.

\& Koch, 1991). We therefore estimated the time constants to see how these varied with mean activity and phase locking. Details of the simulations, measurement of effective connectivity, and derivation of the effective time constants will be found in the appendix.

\section{Results}

We found that increases in the activity level of the network were universally associated with increases in the phase locking between and within the populations as represented by the peak shift predictor subtracted crosscorrelation. This held for large ranges of mean activity with a falloff at very high levels. This was observed regardless of the way that the activity level was varied (e.g., changing the input to population one, varying the number of connections, or manipulating the synaptic efficacies).

First, we used the model incorporating two cortical areas, each comprising three layers (see Figure 1b) and manipulated the input activity level (see Figure 2) to layer 4 of the first area. Phase locking rose systematically with activity levels, with a falloff at very high levels.

The second component of our study represented an exploration of a larger parameter space, using the second model consisting of two areas, each comprising 100 cells. Figure 3 shows the phase locking between the 
two populations as a function of mean activity in population 1 , for 10 different levels of extrinsic connectivity. In these simulations, the input activity level was varied systematically to elicit changes in the dynamics. It can be seen in Figures $3 \mathrm{a}$ to $3 \mathrm{~d}$ that phase locking increases monotonically between the spike trains or local field potential, as the activity level increases. Furthermore, the rate of increase of phase locking with mean activity increases with extrinsic connectivity. This is expressed as an increase in the slope of the regression of phase locking on mean activity and represents an interaction between mean activity and extrinsic connectivity in producing synchronization. Figures $3 e$ and $3 f$ illustrate the spiking and subthreshold activity in populations 1 and 2 at low and high levels of activity, respectively. It is seen that as activity rises, the spiking activity in each population becomes increasingly oscillatory.

In the previous simulations, changes in the dynamics were elicited under different levels of extrinsic connectivity by manipulating the input to population 1 . The results pointed to an interaction between input activity and extrinsic connectivity. To characterize these influences fully, we examined the main effect of connectivity per se on synchrony by changing both extrinsic and intrinsic connections. This can be regarded as an analysis of the relationships between synaptic efficacy or anatomical connectivity and functional connectivity. Figure 4 shows plots of phase locking between spike trains for the second model, when the input activity level was kept constant and the interarea connectivity level, interarea weights, and intra-area weights were manipulated respectively (i.e., the density or efficacy of connections were modulated). These simulations were performed with feedback influences mediated either by AMPA or NMDA receptors. As shown in the figure, the phase locking, within and between populations, increases to a certain level before reaching a plateau and eventually decreasing slightly, as either the extrinsic or intrinsic connectivity level increases (through changing the number of connections or weight values).

Figure 3: Facing page. Synchrony versus mean activity for the second model. (a, b) The peak shift predictor subtracted cross-correlation between the time series of number of cells spiking per ms for each population is plotted against mean number of cells spiking in population 1 per millisecond for extrinsic reciprocal connectivities of (a) $5 \%, 15 \%, 45 \%, 65 \%$ and (b) $75 \%, 85 \%$, and $95 \%$. (b, c) The peak cross-correlation between the time series of mean membrane potential is plotted against mean membrane potential of population 1 for the same extrinsic connectivities as in a and b. (e, f) The spiking activity in populations 1 and 2 are plotted over the course of 2 seconds. Time is plotted horizontally, and all 100 neurons are shown on the vertical axis. The membrane potential is shown in terms of the color (see the color scale at the side of the graph). (e, f) For lowand high-input activity levels, respectively. 
A

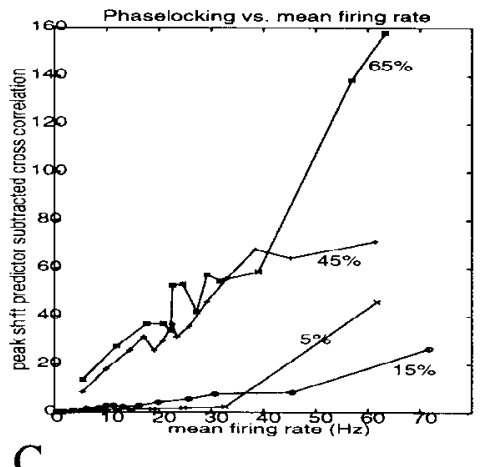

C

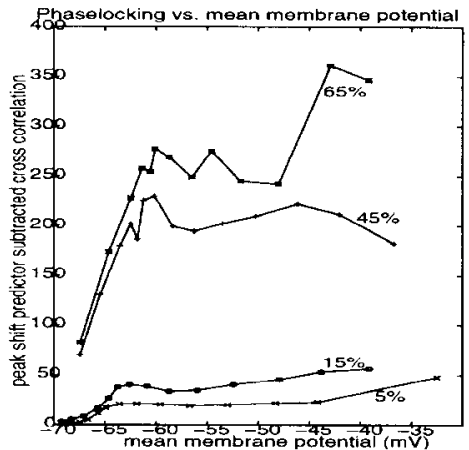

$\mathrm{E}$

20 plot showing spiking activily in pooulation one with a bow input actsity level

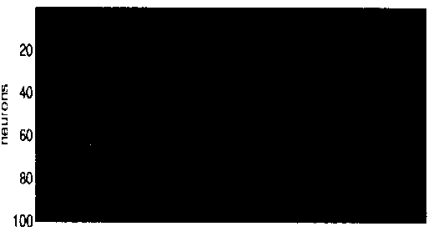

$\begin{array}{llllllllll}200 & 400 & 600 & 800 & 1000 & 1200 & 1400 & 1600 & 1800 & 2000\end{array}$ time (ms)

20 plot showing spiking activity in population two with a low input actinty level

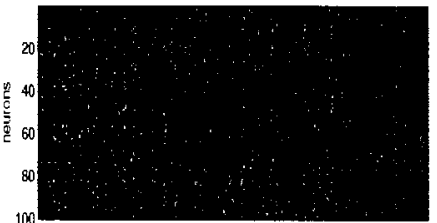

$\begin{array}{lllllllllll}200 & 400 & 600 & 800 & 1000 & 1200 & 1400 & 1600 & 1800 & 2000\end{array}$
B

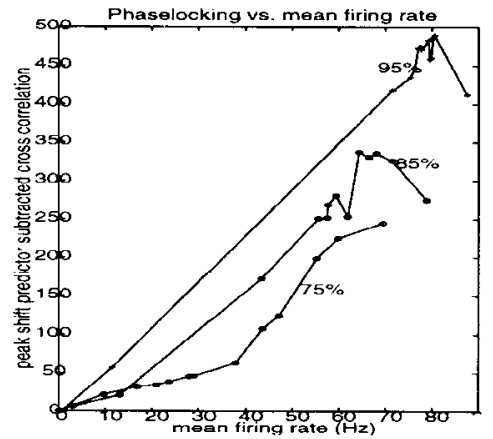

D

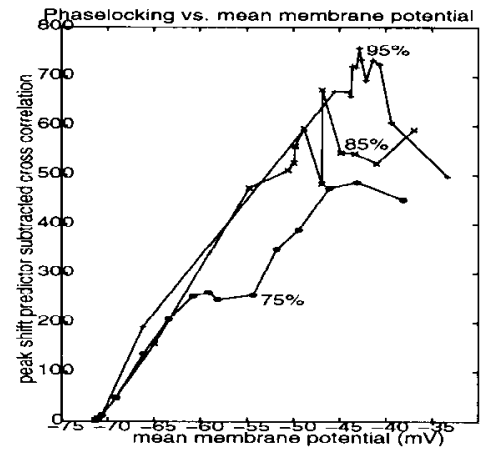

$\mathrm{F}$

20 plot showing spiking actividy in population one with a hight input actovity level

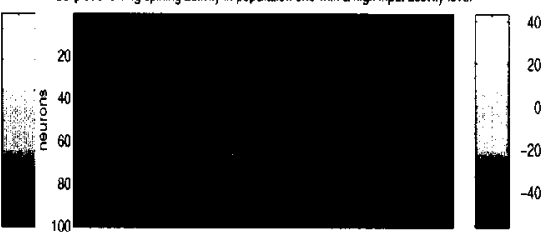

$200 \quad 400 \quad 600 \quad 800 \quad 1000 \quad 1200 \quad 1400 \quad 1600 \quad 1800 \quad 2000$ 20 plol showng spikng actwity in populalion wwo with a high inpul activity level

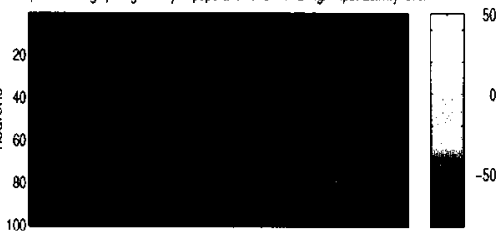

$\begin{array}{llllllllll}200 & 400 & 600 & 800 & 1000 & 1200 & 1400 & 1600 & 1800 & 2000\end{array}$ 
Next, we increased the extrinsic mean transmission delays from 5 to $8 \mathrm{~ms}$. This was done to simulate longer-range connections and assess their effect on the behavior of phase locking with activity level. Figure 5 shows plots of phase locking (within and between populations) against activity level varied in four different ways using AMPA or NMDA feedback connections. As can be seen in Figure 5a, the results are almost identical to those of Figure 3a, indicating that increasing the transmission delay does not significantly alter the nature of the phase locking. Figures $5 \mathrm{c}$ and $5 \mathrm{~d}$ show the phase locking between one neuron in population 1 and the rest of the population. These results suggest that phase locking varies with activity level in much the same way as between populations. Figures $5 \mathrm{~b}$ and $5 \mathrm{~d}$ show that changing the receptor types to NMDA does not have a significant effect on how phase locking varies with activity.

Figure 6 shows the relationship between phase locking and mean firing rate when the input to area 1 is changed systmatically under different levels of inhibition. The level of inhibition was manipulated by changing either the proportion of inhibitory neurons (see Figure 6a) or the value of the inhibitory synaptic time constants (see Figure $6 \mathrm{~b}$ ). Under all levels of inhibition within the network, a monotonic relationship between phase locking and mean activity was evidenced. As inhibition increased, the rate of increase of phase locking with mean activity decreased. This was evident as a decrease in the slope of the regression of phase locking on mean activity. These results

Figure 4: Facing page. Synchrony as a function of connectivity for the second model. $(a, b)$ The level of intrinsic connectivity was held constant at $90 \%$, while the extrinsic connectivity was varied through $5 \%, 15 \%, 25 \%, 35 \%, 45 \%, 55 \%$, $65 \%, 75 \%, 85 \%$, and $95 \%$. Plotted horizontally is the level of extrinsic connectivity. Plotted vertically is the maximum value of the shift predictor subtracted cross-correlation between the two neuronal populations or within population 1. (a) The peak cross-correlation between the time series of number of cells spiking per ms for each population is plotted against extrinsic connectivity. The two cases when the feedback receptors were AMPA and NMDA are shown. (b) The peak cross-correlation between the time series of spikes per millisecond in one cell and the spikes per millisecond in the rest of population 1 is plotted against the percentage of extrinsic connectivity. Again, this graph shows this plot under both AMPA and NMDA feedback receptors. (c, d) Same as $a$ and $b$, except that here the number of connections was not changed. Instead, the actual values of the extrinsic weights were varied with the density of extrinsic connections remaining at $5 \%$. Here, extrinsic synaptic weight is plotted horizontally. (e) Intrinsic and extrinsic connectivity levels remained constant $(90 \%$ and $5 \%$, respectively), while intrinsic weights were increased. This plot shows how phase locking varies between populations and also within each population as the intrinsic weights are increased. These graphs show the results for AMPA feedback receptors, but similar findings were obtained with NMDA feedback receptors. 
A

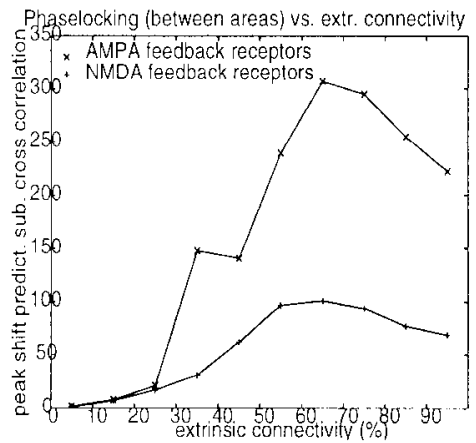

$\mathrm{C}$

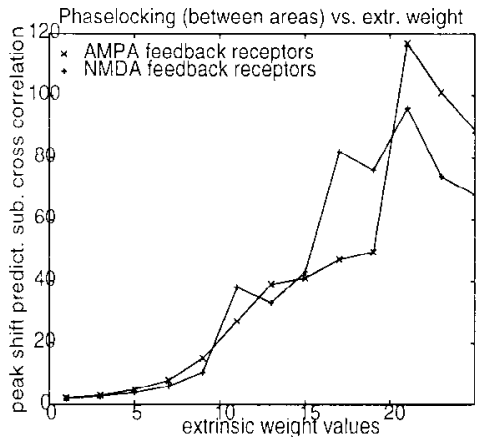

$\mathrm{E}$

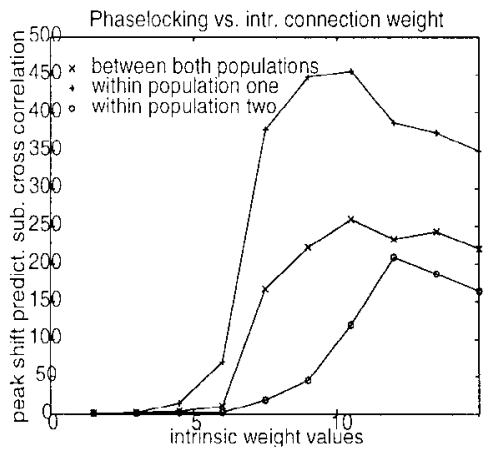

\section{B}

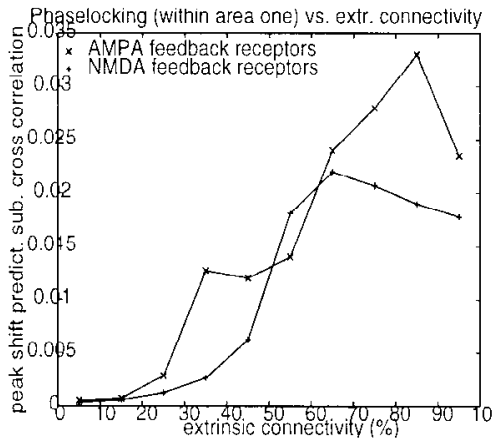

\section{D}

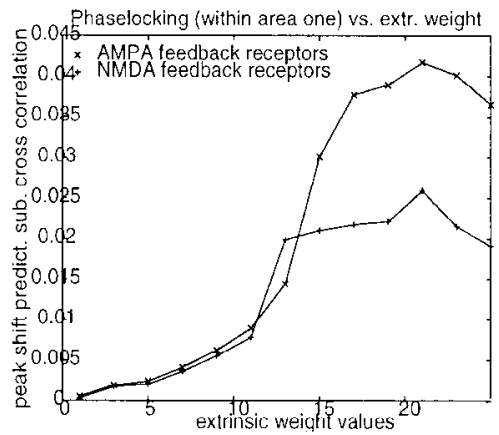


A

B

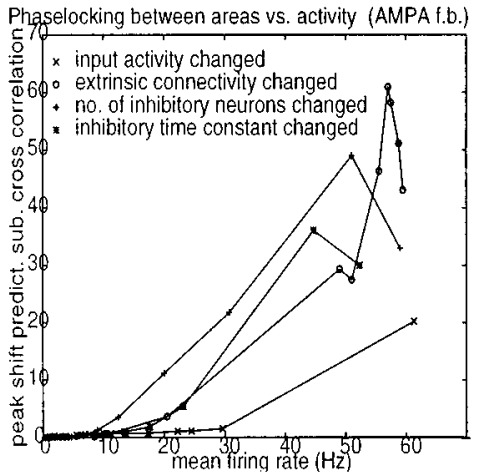

$\mathrm{C}$

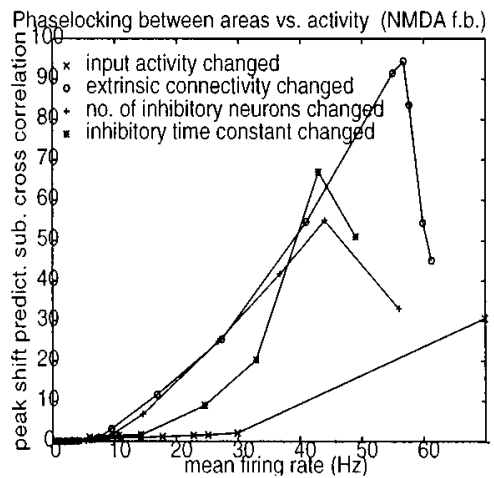

D
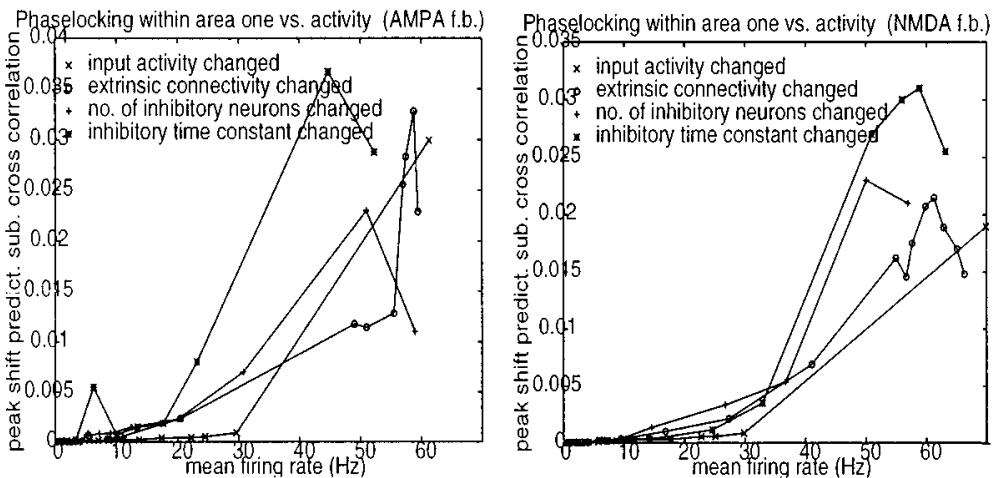

Figure 5: These graphs show how phase locking varies with neuronal activity when the extrinsic delays were increased to a mean of $8 \mathrm{~ms}$. Here, the activity level was varied in four different ways: (1) By changing the input activity levels while all other parameters remained constant. The effect of this manipulation on phase locking and activity level is denoted by x. (2) By varying the extrinsic connectivity level between $5 \%$ and $95 \%$ (These data are shown by $\circ$ ). (3) By changing the proportion of inhibitory neurons between $60 \%$ to $0 \%$. This is denoted by + . (4) By changing the values of the inhibitory synaptic time constants from 500 to $0.5 \mathrm{~ms}$ (denoted by ${ }^{*}$ ). $(\mathrm{a}, \mathrm{c})$ The feedback receptors were AMPA. $(b, d)$ Feedback receptors were NMDA. $(a, b)$ Phase locking against mean firing rate between populations. (c, d) Phase locking between the firing rates of one cell and the rest of the population.

point to a clear interaction between input activity and inhibition level, where inhibition attenuates the increase in synchrony with mean activity. 
A

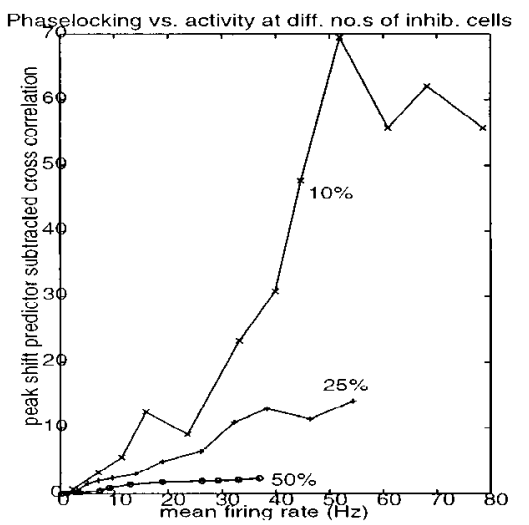

$\mathrm{B}$

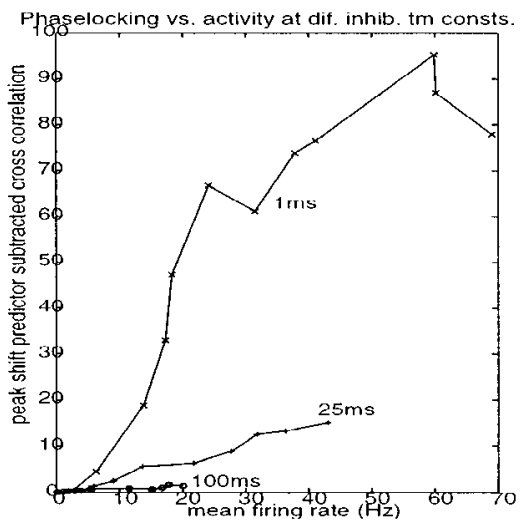

Figure 6: (a) Phase locking versus mean firing rate as input to area 1 is varied systematically with network inhibitory cell proportions of $10 \%, 25 \%$, and $50 \%$. (b) Is the same as $a$ except inhibition is varied by changing the inhibitory synaptic time constants between 1,25, and $100 \mathrm{~ms}$ while keeping the number of inhibitory cells constant. The feedback receptors were AMPA in both cases.

To address the mechanisms behind the relationship between activity and phase locking, we assessed how the effective connectivity and mean instantaneous membrane time constants varied with both activity level and phase locking. The results of this analysis are shown in Figure 7. Figures 7a and $7 \mathrm{~b}$ show how the effective connectivity varies with mean firing rate (see Figure 7a) and with phase-locking (see Figure 7b), as the input activity level was manipulated. A saturating relationship was observed with a falloff at very high levels. Figures $7 \mathrm{c}$ and $7 \mathrm{~d}$ show the relationship between the mean membrane time constant and mean firing rate (see Figure 7c) and between the mean membrane time constant and phase locking (see Figure 7d). As mean firing rate increases, the mean membrane time constant decreases (see Figure 7c). The decrease in mean membrane time constant is accompanied by an increase in both synchrony and effective connectivity between the simulated populations. The implications of this finding are discussed below.

\section{Discussion}

Our results suggest that the phenomenon of phase locking's increasing with activity level is a robust effect that is relatively insensitive to the context in which the activity level is varied, changes in the transmission delays, the 
A $\quad$ B

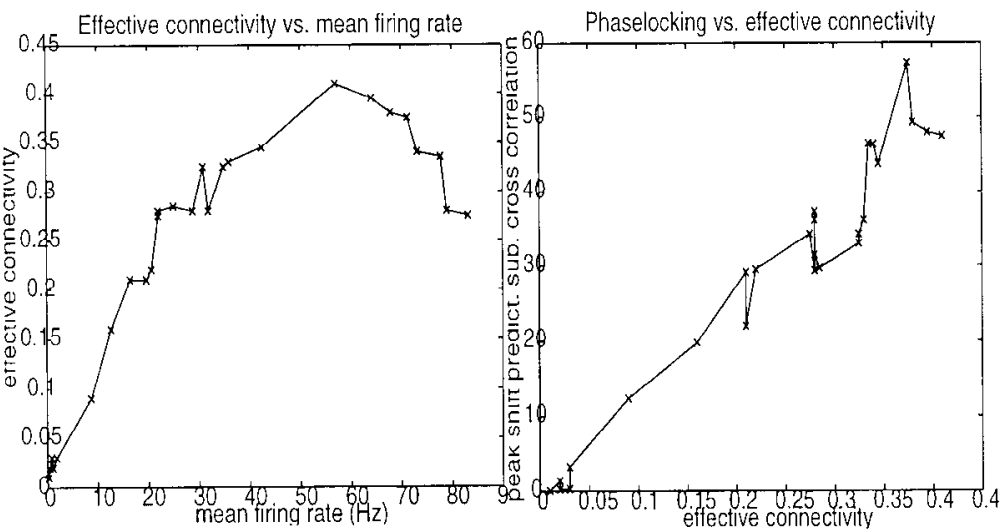

$\mathrm{C}$

\section{D}
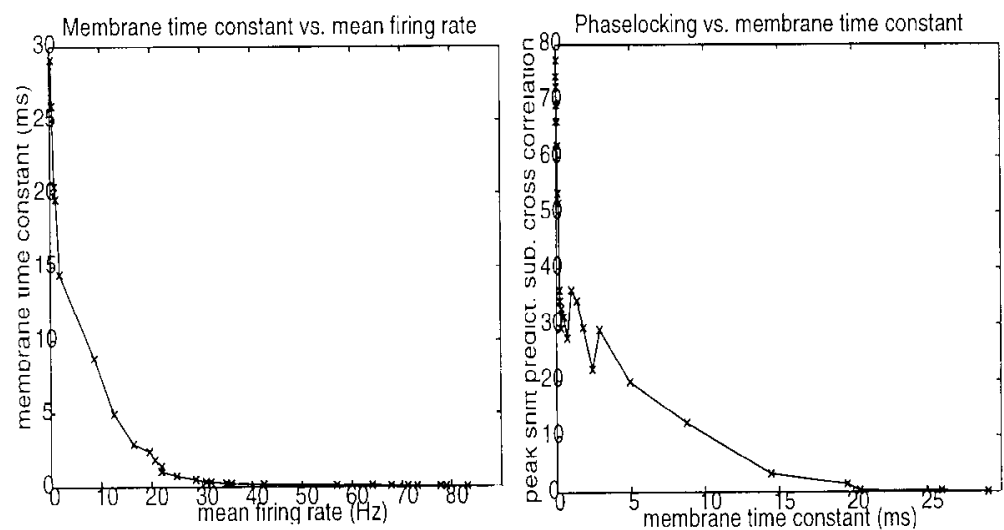

Figure 7: (a) Effective connectivity between the two populations of the second model (as given by the average probability of a cell in population one causing a connected cell in population two to fire) is plotted against average firing rate. The extrinsic connectivity was $25 \%$, and the mean firing rate was manipulated by varying the input activity. (b) A graph of functional connectivity as given by the peak shift predictor subtracted cross-correlation in terms of effective connectivity. (c) A plot of the mean membrane time constant, computed for each activity level, against mean firing rate. (d) A plot of phase locking as a function of the mean membrane time constant.

type of synapse, the number of cells, and the laminar structure within the populations. They also show that functional connectivity (i.e., synchrony) 
varies with mean activity in much the same way as effective connectivity and that there is an almost monotonic relationship between the two metrics (see Figure $7 \mathrm{~b}$ ). These results clearly hold only for the simulations presented, which addressed unstructured, continuous, or stationary dynamics. However, it may be reasonable to generalize the inference to real neuronal populations with similar simple architectures if they are expressing relatively stationary dynamics.

4.1 Activity Levels and Effective Connectivity. This work indirectly addresses the relationship between rate and synchrony coding and suggests that they may represent two perspectives on the same underlying dynamic. In this view, synchronized, mutually entrained signals enhance overall firing levels and can be thought of as mediating an increase in the effective connectivity between the two areas. Equivalently, high levels of discharge rates increase the effective connectivity between two populations and augment the fast synchronous exchange of signals. In a previous modeling study, Aertsen \& Preissl (1990) showed that by increasing the level of network activity, the efficacy of the effective synaptic connections increases: "The efficacy varies strongly with pool activity, even though the synapse itself is kept at a fixed strength throughout all simulations. With increasing pool activity, the efficacy of the connection initially increases strongly to reach a maximum, after which it slowly decays again." This result is consistent with our findings (see Figure 7a) and is intuitive; as the network activity is increased, the individual neuronal connections come into play more. This can be explained in the following way: If network activity is very low, the inputs to a single neuron (say neuron $j$ ) will cause only a subthreshold excitatory postsynaptic potential (EPSP) in neuron $j$. If some presynaptic neuron (say neuron $i$ ) fires, so that it provides input to neuron $j$, this input will be insufficient to cause neuron $j$ to fire. However, if the pool activity is high enough to maintain a slightly subthreshold EPSP in neuron $j$, then an input from neuron $i$ is more likely to push the membrane potential of neuron $j$ over the threshold and elicit an action potential. This effect resembles the phenomenon of stochastic resonance (Wiesenfeld \& Moss, 1995). As pool activity becomes very large, however, the coincident input to cell $j$ will eventually become enough to make neuron $j$ fire without any input from cell $i$, thus decreasing the influence that cell $i$ has on cell $j$ and consequently the effective connectivity between the two cells. This may explain the slow decline in effective connectivity as the network activity becomes very large (see Figure 7a). In short, we can say that the pool activity provides a background neuronal tonus that, depending on its magnitude, will make activity in neuron $i$ more or less viable in eliciting activity in neuron $j$.

4.2 Activity Levels and Synchronization. The above argument pertains to the relationship between mean activity and effective connectivity but does not deal explicitly with the relationship between activity levels and 
synchronization. This study examined the mechanistic basis of synchronized and oscillatory dynamics at high levels of activity. The membrane time constants were shown to decrease with mean activity, and thus synchrony emerged with shorter membrane time constants. The decrease in time constants is a natural consequence of conjointly increasing membrane conductances through excitatory and inhibitory channels at high levels of activity (see the appendix). Hence, as activity levels increase, smaller membrane time constants promote the synchronous gain in the network; that is, individual neurons became more sensitive to temporal coincidences in their synaptic inputs, responding with a higher firing rate to synchronous rather than asynchronous inputs. In other words, as the level of activity increases, network interactions tend toward synchronous firing. At the same time, the overall increase in background synaptic activity causes individual cell membranes to become more leaky, thereby decreasing their effective time constants (Bernander et al., 1991). This promotes synchrony by increasing the sensitivity of individual cells to synchronous inputs. Put simply, there is a circular causality: Only synchronous interactions can maintain high firing rates when temporal integration is reduced. High firing rates reduce temporal integration. This behavior underlies the emergence of self-selecting dynamics in which high degrees of synchrony can be both cause and consequence of increased activity levels.

In our model architecture, extrinsic excitatory connections targeted both excitatory and inhibitory neurons within the population. Further simulations are clearly needed to determine if the relative proportion of excitatory targets is an important parameter in relation to the phenomena that we have observed. One conjecture, however, is that it is not the overall excitation or inhibition elicited by afferent input that determines the dynamics, but rather the increase in membrane conductance consequent upon the conjoint increase in balanced excitatory and inhibitory activity. In other words, driving predominantly inhibitory subpopulations will inhibit excitatory cells, or driving excitatory cells will excite inhibitory cells. In both cases, the overall level of excitatory and inhibitory presynaptic discharges will reduce the effective membrane time constants and predispose the population to fast dynamic and synchronized dynamics.

4.3 Uncoupling of Activity and Synchronization. The overall impression given by our results is that there is an obligatory relationship between mean activity and synchronized interactions. This is mediated by decreases in the effective membrane time constants under high levels of activity. Due to the reduced capacity for temporal integration, the only dynamics that can ensue are synchronous ones. It is important, however, to qualify this conclusion by noting that in this study, the inputs driving the coupled neuronal populations were spatiotemporally unstructured and continuous. Clearly, desynchronization between two dynamic cell assemblies is not only a possibility but can be observed in both the real brain and simulations where 
changes in synchrony have, in some instances, been found to occur without any change in mean firing rate. Such regional decoupling of spike timing and firing rates has been reported in primary sensory cortices (Roelfsema, Konig, Engel, Sireteanu, \& Singer, 1994; deCharms \& Merzenich, 1996; Fries, Roelfsma, Engel, Konig, \& Singer, 1997) and may reflect feedback influences from higher cortical areas (Lumer et al., 1997b). Our input stimulus consisted of unstructured random noise that did not have any spatiotemporal structure. Furthermore, our models did not include any feature selectivity (such as orientation columns). It is this feature specificity and stimulus structure that may cause a regional decoupling of synchrony and firing rate. This decoupling could specify which neuronal populations are excluded from dynamic cell assemblies coding for the feature in question. It could be that the temporal patterning of action potentials in primary areas, which show a regional decoupling between synchrony and firing rate, may lead to changes in firing rates in the areas that they target, and thus such changes in synchrony will be reflected in changes in global activity levels (i.e., summed over all dynamic cell assemblies), if not local activity levels. In other words, a particular population could maintain high levels of desynchronized activity, in relation to its inputs from one cell assembly, if it was part of another dynamic cell assembly that did exhibit a coupling between overall activity and synchrony.

In essence, although the coupling that we have shown between mean activity and synchronization may represent a generic property of cortical dynamics, it should be noted that desynchronized interactions can arise from nonlinear coupling of a stronger sort than that employed in our current model or by specific inputs that selectively engage distinct cohorts of interacting populations. Other mechanisms that may cause synchrony to decouple from firing rates include those that are capable of modulating firing rates as synchrony increases, such as fast synaptic changes. However, in the context of our studies that looked explicitly at stationary dynamics, this is unlikely to be an explanatory factor. These and other parameters have to be explored before any definitive statements can be made about the relationship between mean activity and synchronization in a real-world setting. However, our results point to some fundamental aspects of neural interactions under a set of minimal assumptions. Our current lines of inquiry include revisiting the relationship between mean activity and synchrony in the context of evoked transients (Chawla, in press) and trying to characterize the nonlinear coupling between neuronal populations that underpins asynchronous interactions (Friston, 1997).

4.4 Practical Implications. The final point that can be made on the basis of our findings relates to macroscopic measures of neural activity such as those used in functional brain imaging. Functional magnetic resonance imaging (FMRI) and positron emission tomography (PET) have been established as tools for localizing brain activity in particular tasks using the 
blood oxygenation level-dependent response (BOLD signal in FMRI) and blood flow (PET). The fMRI BOLD signal is attributed to changes in local venous blood deoxygenation. These studies rely on the assumption that such changes are representative of global synaptic activity levels. This is supported by optical imaging studies (Frostig, Lieke, Ts'o, \& Grinvald, 1990) showing that there is a local coupling between neuronal activity integrated over a few seconds and the microcirculation (hemodynamics). The lack of temporal sensitivity of fMRI raises the possibility that such measurements will fail to identify areas in which neuronal processes are expressed solely in terms of changes in synchrony. However, this study demonstrates a clear link between mean firing rates and synchronization, suggesting that metrics based on mean synaptic activity may in part be sensitive to changes in synchronization. We are investigating this issue empirically, using combined fMRI and electroencephalograms and with simulations looking at evoked transients and dynamic correlations.

\section{Appendix A: Modeling Neuronal Dynamics}

A.1 Model 1. The instantaneous change in membrane potential of each model neuron, $V(t)$, was given by:

$$
\tau_{m} d V / d t=-V+V_{0}-\Sigma_{j} g_{j}\left(V-V_{j}\right)
$$

where $\tau_{m}$ is a passive membrane time constant set at $16 \mathrm{~ms}(8 \mathrm{~ms})$ for cortical excitatory (inhibitory) cells and the sum on the right-hand side is over synaptic currents. $V_{0}$ denotes the passive resting potential that was set to a value of $-60 \mathrm{mV}$. $V_{j}$ are the equilibrium potentials for the $j$ th synaptic type. $V$ was reset to the potassium reversal potential of $-90 \mathrm{mV}$ when it exceeded a threshold of $-50 \mathrm{mV}$ and a spike event was generated for that unit. Synaptic activations of AMPA, GABAa, and GABAb receptors were expressed as a change in the appropriate channel conductance, $g_{j}$, according to a dual exponential response to single-spike events in afferent neurons given by:

$$
g=g_{\text {peak }}\left[\exp \left(-t / \tau_{1}\right)-\exp \left(-t / \tau_{2}\right)\right] /\left[\exp \left(-t_{\text {peak }} / \tau_{1}\right)-\exp \left(-t_{\text {peak }} / \tau_{2}\right)\right]
$$

$\tau_{1}$ and $\tau_{2}$ are the rise and decay time constants, respectively, and $t_{\text {peak }}$, the time to peak. $t_{\text {peak }}=\tau_{1} \tau_{2} /\left(\tau_{1}-\tau_{2}\right)$. g peak represents the maximum conductance for any particular receptor. Conductances were implicitly normalized by a leak membrane conductance, so that they were adimensional. The implementation of NMDA channel, was based on Traub, Wong, Miles, \& Michelson, (1991):

$$
\begin{aligned}
& I_{N M D A}=g_{N M D A}(t) M\left(V-V_{N M D A}\right) \\
& d g_{N M D A} / d t=-g_{N M D A} / \tau_{2} \\
& M=1 /\left(1+\left(\mathrm{Mg}^{2+} / 3\right)(\exp [-0.07(V-\xi)])\right.
\end{aligned}
$$


Table 1: Parameter Values of Model 1.

\begin{tabular}{lllll}
\hline Receptor & $g_{\text {peak }}(\mathrm{mS})$ & $\tau_{1}(\mathrm{~ms})$ & $\tau_{2}(\mathrm{~ms})$ & $V_{j}(\mathrm{mV})$ \\
\hline AMPA & 0.05 & 0.5 & 2.4 & 0 \\
GABAa & 0.175 & 1 & 7 & -70 \\
GABAb & 0.0017 & $30-90$ & $170-230$ & -90 \\
NMDA & 0.01 & 0 & 100 & 0 \\
\hline
\end{tabular}

$I_{N M D A}$ is the current that enters linearly into the equation for $d V / d t$, above. $g_{N M D A}$ is a ligand-gated virtual conductance. $M$ is a modulatory term that mimicks the voltage-dependent affinity of the $\mathrm{Mg}^{2+}$ channel pore. $\xi$ is $-10 \mathrm{mV}$ and $\mathrm{Mg}^{2+}$ is the external concentration of $\mathrm{Mg}^{2+}$ often used in hippocampal slice experiments $(2 \mathrm{mM})$. These and other parameters (see Table 1) were consistent with experimental data (see Lumer et al., 1997a, for details).

A.2 Mode1 2. Model 2 was similar to model 1 but included explicit modeling of $\mathrm{Na}^{+}$and $\mathrm{K}^{+}$channels that mediate action potentials. The neuronal dynamics of this model were based on the equations from the Yamada, Koch, and Adams (1989) single neuron model, using the Hodgkin and Huxley formalism:

$$
\begin{array}{rlrl}
d V / d t= & -1 / C_{M}\left\{\left(g_{\mathrm{Na}} m^{2} h\left(V-V_{\mathrm{Na}}\right)+g_{K} n^{2} y\left(V-V_{K}\right)+g_{l}\left(V-V_{l}\right)\right.\right. \\
& \left.+g_{A M P A}\left(V-V_{A M P A}\right)+g_{G A B A}\left(V-V_{G A B A}\right)\right\}, \\
d m / d t= & \alpha_{m}(1-m)-\beta_{m} m, & d h / d t=\alpha_{h}(1-h)-\beta_{h} h, \\
d n / d t= & \alpha_{n}(1-n)-\beta_{n} n, & & d y / d t=\alpha_{y}(1-y)-\beta_{y} y, \\
d g_{A M P A} / d t=-g_{A M P A} / \tau_{A M P A} & d g_{G A B A} / d t=-g_{G A B A} / \tau_{G A B A}
\end{array}
$$

$C_{M}$ represents the membrane capacitance $(1 \mu \mathrm{F}), g_{\mathrm{Na}}, g_{K}$ and $g_{l}$ represent the maximum $\mathrm{Na}+$ channel, $\mathrm{K}^{+}$channel and leakage conductances respectively. $V_{\mathrm{Na}}$ represents the $\mathrm{Na}+$ equilibrium potential and similarly for $\mathrm{V}_{\mathrm{K}}$ and $\mathrm{V}_{l} . m$, $h, n$, and $y$ are the fraction of $\mathrm{Na}^{+}$and $\mathrm{K}^{+}$channel gates that are open. $g_{A M P A}$ and $g_{G A B A}$ are the conductances of the excitatory (AMPA) and inhibitory (GABAa) synaptic channels, respectively. $\tau$ represents the excitatory and inhibitory decay time constants. $\alpha_{n}, \beta_{n}, \alpha_{m}, \beta_{m}, \alpha_{h}, \beta_{h}, \alpha_{y}, \beta_{y}$ are nonnegative functions of $V$ that model voltage-dependent rates of channel configuration transitions. Specific values for the parameters of this model are given in Table 2.

A.3 Measuring the Effective Connectivity. Consider two cells-the first, cell $i$, being some neuron in population 1 and the second, cell $j$, being in population 2, that receives an input from cell $i$. The number of times cell $j$ fires in a time window of $10 \mathrm{~ms}$ immediately following an event in cell $i$ is $n_{j}$. The total number of spikes from cell $i$ is $n_{i} . n_{j} / n_{i}$ is an estimate of the con- 
Table 2: Parameter Values of Model 2.

\begin{tabular}{llll}
\hline Receptor/Channel & $g_{\text {peak }}(\mathrm{mS})$ & $\tau(\mathrm{ms})$ & $V_{j}(\mathrm{mV})$ \\
\hline AMPA & 0.05 & 3 & 0 \\
GABAa & 0.175 & 7 & -70 \\
$\mathrm{Na}^{+}$ & 200 & & 50 \\
$\mathrm{~K}^{+}$ & 170 & & -90 \\
Leak & 1 & -60 \\
\hline
\end{tabular}

ditional probability that cell $j$ fires in a time interval after cell $i$. To discount the effect of incidental firing in cell $j$, we subtracted the probability that cell $j$ would fire spontaneously in this interval $(p)$ when cell $i$ had not previously fired. This was calculated as the total number of spikes from cell $j$ divided by the total number of $10 \mathrm{~ms}$ intervals comprising the time series (having discounted intervals following an input from cell $i$ ). The resulting estimate can be construed as an index of effective connectivity, $E=n_{j} / n_{i}-p$.

A.4 Determining the Effective Membrane Time Constant. The effective membrane time constant was determined as follows: $\tau_{\mathrm{mem}}=R_{m} C_{m}$, where $R_{m}$ is the membrane resistance and:

$$
\begin{aligned}
C_{m} d V / d t= & g_{l}\left(V-V_{l}\right)+g_{A M P A}\left(V-V_{A M P A}\right)+g_{G A B A}\left(V-V_{G A B A}\right) \\
& + \text { sodium and potassium currents. }
\end{aligned}
$$

Discounting the internal sodium and potassium channel dynamics that generate the action potentials, the last equation can be rearranged in the following way;

$$
\begin{aligned}
C_{m} d V / d t= & \left(g_{l}+g_{A M P A}+g_{G A B A}\right)\left(V-V_{0}\right)+g_{A M P A}\left(V_{0}-V_{A M P A}\right) \\
& +g_{G A B A}\left(V_{0}-V_{G A B A}\right)+g_{l}\left(V_{0}-V_{l}\right) .
\end{aligned}
$$

$V_{0}$ denotes the resting membrane potential. Over time, the average currents (inhibitory, excitatory and leakage) cancel each other out. Therefore, $g_{A M P A}\left(V_{0}-V_{A M P A}\right)+g_{G A B A}\left(V_{0}-V_{G A B A}\right)+g_{l}\left(V_{0}-V_{l}\right)$ is negligible compared to $\left(g_{l}+g_{A M P A}+g_{G A B A}\right)\left(V-V_{0}\right)$ and, thus approximately, $\tau_{\text {mem }}=$ $C_{m} /\left(g_{l}+g_{A M P A}+g_{G A B A}\right)$ at any given time for any particular cell. In this article, we take the average value of $\tau_{\text {mem }}$ over time and units.

\section{Acknowledgments}

This work was supported by the Wellcome Trust. 


\section{References}

Abeles, M. (1982). Role of the cortical neuron: Integrator or coincidence detector? Isr J Med Sci, 18, 83-92.

Aertsen, A., \& Preissl, H. (1990). Dynamics of activity and connectivity in physiological neuronal networks. In W. G. Schuster (Ed.), Nonlinear dynamics and neuronal networks (pp. 281-302). New York: VCH Publishers.

Ahmed, B., Anderson, J., Douglas, R., Martin, K., \& Nelson, J. (1994). Polyneuronal innervation of spiny stellate neurons in cat visual cortex. J. Comp. Neurol., 341, 39-40.

Beaulieu, C., \& Colonnier, M. (1983). The number of neurons in the different laminae of the binocular and monocular regions of area 17 in the cat. J. Comp. Neurology, 217, 337-344.

Beaulieu, C., \& Colonnier, M. (1985). A laminar analysis of the number of roundasmmetrical and flat-symmetrical synapses on spines, dendritic trunks and cell bodies in area 17 of the cat. J. Comp. Neurology, 231, 180-189.

Beaulieu, C., Kisvarday, Z., Somogyi, P., \& Cynader, M., (1992). Quantitative distribution of GABA-immunopositive and -immunonegative neurons and and synapses in the monkey striate cortex (area 17). Cerebral Cortex, 2, 295309.

Bernander, O., Douglas, R. J., Martin, K. A. C., \& Koch, C. (1991). Synaptic background activity influences spatiotemporal integration in single pyramidal cells. Proc. Natl. Acad. Sci. USA, 88, 11569-11573.

Calabresi, P., Mercuri, N. B., Stefani, A., \& Bernardi, G. (1990). Synaptic and intrinsic control of membrane excitability of neostriatal neurons. I. An in vivo analysis. J. Neurophysiol., 63(2), 651-662.

Chawla, D. (forthcoming). Relating macroscopic measures of brain activity to fast dynamic neuronal interactions. Neural Computation.

Conde, F., Lund, J., Jacobwitz, D., Baimbridge, K. G., \& Lewis, D. (1994). Local circuit neurons immunoreactive for calretin, (albindin $D=28$ ) or parvalbumin in monkey prefrontal cortex: Distribution and morphology. J. Neurosci., 341, 95-116.

deCharms, R. C., \& Merzenich, M. M. (1996). Primary cortical representation of sounds by the coordination of action potential timing. Nature, 381, 610-613.

Domenici, L., Harding, G. W., \& Burkhalter, A. (1996). Patterns of synaptic activity in forward and feedback pathways within rat visual cortex. J. Neurophysiol., 74, 2649-2664.

Engel, A. K., Konig, P., Kreiter, A. K., Gray, C. M., \& Singer, W., (1990). Temporal coding by coherent oscillations as a potential solution to the binding problem. in H. G. Schuster (Ed.), Nonlinear dynamics and neural networks. New York: VCH Publishers.

Engel A. K., Konig, P., Kreiter, A. K., \& Singer, W. (1991). Interhemispheric synchronization of oscillatory neuronal responses in cat visual cortex. Science, 252, 1177-1179.

Felleman, D. J., \& VanEssen, D. C. (1991). Distributed hierarchical processing in the primate cerebral cortex. Cerebral Cortex, 1, 1-47. 
Fries, P., Roelfsema, P. R., Engel, A., Konig, P., \& Singer, W. (1997). Synchronization of oscillatory responses in visual cortex correlates with perception in interocular rivalry. Pro Natl Acad Sci USA, 94,12699-12704.

Freiwald, W. A., Kreiter, A. K., \& Singer, W. (1995). Stimulus dependent intercolumnar synchronization of single unit responses in cat area 17. Neuroreport, $6,2348-2352$.

Friston, K. J. (1994). Functional and effective connectivity in neuroimaging: A synthesis. Human Brain Mapping, 2, 56-78.

Friston, K. J. (1997). Transients, metastability and neuronal dynamics. NeuroImage, 5, 164-171.

Frostig, R. D., Lieke, E. E., Ts'o, D. Y., \& Grinvald, A. (1990). Cortical functional architecture and local coupling between neuronal activity and the microcirculation revealed by in vivo high-resolution optical imaging of intrinsic signals. Proc. Natl. Acad. Sci. USA, 87, 6082-6086.

Gray, C. M., Konig, P., Engel, A. K., \& Singer, W. (1989). Oscillatory responses in cat visual cortex exhibit inter-columnar synchronization which reflects global stimulus properties, Nature, 338, 334-337.

Gray, C. M., Engel, A. K., Konig, P., \& Singer, W. (1990). Temporal properties of synchronous oscillatory neuronal interactions in cat striate cortex, In W. G. Schuster (Ed.), Nonlinear dynamics and neural networks. New York: VCH Publishers.

Johnson, R. R., \& Burkhalter, A., (1996). Microcircuitry of forward and feedback connections within rat visual cortex. J. Physiol., 160, 106-154.

Kawaguchi, Y. (1995). Physiological subgroups of nonpyramidal cells with specific morphological characteristics in layer ii/iii of rat frontal cortex. J. Neurosci., 15, 2638-2655.

Konig, P., Engel, A. K., \& Singer, W. (1995). Relation between oscillatory activity and long-range synchronization in cat visual cortex. Proc. Natl. Acad. Sci. USA, 92, 290-294.

Lorenzon, N. M., \& Foehring, R. C. (1992). Relationship between repetitive firing and afterhyperpolarizations in human neocortical neurons. J. Neurophysiol., 67(2), 350-363.

Lumer, E. D., Edelman, G. M., \& Tononi, G. (1997a). Neural dynamics in a model of the thalamocortical system I. Layers, loops and the emergence of fast synchronous rhythms. Cerebral Cortex, 7, 207-227.

Lumer, E. D., Edelman, G. M., Tononi, G. (1997b). Neural dynamics in a model of the thalamocortical system II. The role of neural synchrony tested through perturbations of spike timing. Cerebral Cortex, 7, 228-236.

Milner, P. M. (1974). A model for visual shape recognition. Psychological Review, 81(6), 521-535.

Nowak, L. G., Munk, M. H., Nelson, J. I., James, A. C., \& Bullier, J. (1995). Structural basis of cortical synchronization. I. Three types of interhemispheric coupling. Neurophys., 76, 1-22.

Otis, T., Konick, Y. D., \& Mody, I. (1993). Characterization of synaptically elicited GABAb responses using patch-clamp recordings in rat hippocampal slices. J. Physiol. London, 463, 391-407. 
Otis, T., \& Mody, I. (1992). Differential activation of GABAa and GABAb receptors by spontaneously released transmitter. J. Neurophysiol., 67, 227-235.

Roelfsema, P. R., Engel, A. K., Konig, P., \& Singer, W. (1997). Visuomotor integration is associated with zero time-lag synchronization among cortical areas. Nature, 385, 157-161.

Roelfsema, P. R., Konig, P., Engel, A. K., Sireteanu, R., \& Singer, W. (1994). Reduced synchronization in the visual cortex of cats with strabismic amblyopia. Eur. Journal Neurosci., 6, 1645-1655.

Sporns, O., Tononi, G., \& Edelman, G. M. (1990). Dynamic interactions of neuronal groups and the problem of cortical integration. In W. G. Schuster (Ed.), Nonlinear dynamics and neural networks. New York: VCH Publishers.

Stern, P., Edwards, F., Sakmann, B. (1992). Fast and slow components of unitary EPSCS on stellate cells elicited by focal stimulation in slices of rat visual cortex. J. Physiol. London, 449, 247-278.

Sukov, W., \& Barth, D. S. (1998). Three-dimensional analysis of spontaneous and thalamically evoked gamma oscillations in auditory cortex. J. Neurophysiol., 79(6), 2875-2884.

Traub, R. D., Wong, R. K., Miles, R., \& Michelson, H. (1991). A model of a CA3 hippocampal pyramidal neuron incorporating voltage-clamp data on intrinsic conductances. J. Neurophysiol., 66, 635-650.

von der Malsburg, C. (1981). The correlation theory of the brain (Internal rep.) Max Planck Institute for Biophysical Chemistry, Göttingen, West Germany.

Wiesenfeld, K., \& Moss, W. (1995). Stochastic resonance and the benefits of noise: From ice ages to crayfish and SQUIDs. Nature, 373, 33-36.

Yamada, W. M., Koch, C., \& Adams, P. R. (1989). Multiple Channels and calcium dynamics. In C. Koch \& I. Segev (Eds.), Methods in neuronal modeling, (pp. 97134) Cambridge, MA: MIT Press.

Received March 10, 1998; accepted October 29, 1998. 